\title{
新型不对称苯并噻唑三次甲基菁染料的合成及光稳定性研究
}

\author{
陈秀英* 郭 琳 郑昌戈 高海燕 王海军张 丹 \\ (江南大学食品胶体与生物技术教育部重点实验室＼cjkstart江南大学化学与材料工程学院＼cjkstart无锡 214122)
}

\begin{abstract}
摘要 合成了系列杂环氮原子具有不同取代基的不对称苯并篡唑三次甲基菁染料, 染料结构经过质谱、 ${ }^{1} \mathrm{H} N \mathrm{NR}$ 表征. 测试了染料在乙醇中的吸收和荧光发射光谱, 染料的最大吸收值和苂光发射值分别在 628 631 和 $662 \sim 666 \mathrm{~nm}$ 之间. 染料在溶剂中几乎无荧光，可以极大的降低染料自身的苂光背景干扰. 染料的光降解实验表明：喹啉环上辛烷基取代 和噻唑环上芳基取代时, 染料的光稳定性最强. 染料 $8 \mathbf{a} \sim 8 \mathbf{d}$ 的光降解速率常数分别为 $6.38 \times 10^{-4}, 12.5 \times 10^{-4}, 2.68 \times$ $10^{-4}$ 和 $6.30 \times 10^{-4} \mathrm{~mol} \cdot \mathrm{min}^{-1}$. 循环伏安实验法测试了染料的氧化电位.
\end{abstract}

关键词 苯并噻唑菁染料; 光降解; 循环伏安曲线; 荧光; 氧化电位; 光稳定性

\section{Investigation on Synthesis and Photostabilities of Novel Asymmetric Benzothiazole Trimethine Cyanine Dyes}

\author{
Chen, Xiuying* Guo, Lin Zheng, Changge Gao, Haiyan \\ Wang, Haijun Zhang, Dan
}

(Key Laboratory of Food Colloids and Biotechnology, Ministry of Education, School of Chemical and Material Engineering, Jiangnan University, Wuxi 214122)

\begin{abstract}
Novel asymmetric trimethine benzothiazole cyanine dyes with different $N$-substituents were synthesized and characterized by MS and ${ }^{1} \mathrm{H}$ NMR techniques. The maximums of absorption and emission spectra of the dyes in ethanol were in the range of $628 \sim 631$ and $662 \sim 666 \mathrm{~nm}$, respectively. The dyes were almost non-fluorescent in solvents, which can reduce the background interference greatly. The photodegradation experiment showed that the octyl group on quinoline ring and benzyl group on benzothiazole ring improved the photostabilites of the dyes obviously. The rate constants of photoreaction of the dyes $8 \mathbf{8} \sim 8 \mathbf{d}$ were $6.38 \times 10^{-4}, 12.5 \times 10^{-4}, 2.68 \times 10^{-4}$ and $6.30 \times 10^{-4} \mathrm{~mol} \cdot \mathrm{min}^{-1}$, respectively. Oxidation potentials were determined by cyclic voltammetry.

Keywords benzothiazole cyanine dye; photodegradation; cyclic voltammetry curves; fluorescence; oxidizing potential; photostability
\end{abstract}

菁染料是一种商品化的生物苂光标记用染料, 自从 1856 年 Williams ${ }^{[1]}$ 发现 “豆粉” 蓝菁染料以来, 光导性 能优异的各类菁染料一直受到学者们的广泛关注. 每年 在各种不同的应用领域都有大量的文献相继报道. 如: 用作感光胶片的卤化银乳剂的增感剂及无机半导体材 料 ${ }^{[2 \sim 4]}$ 、光盘的记录介质 ${ }^{[5]}$ 、太阳能电池光敏剂 ${ }^{[6,7]}$ 、激

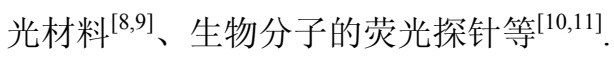

菁染料一般含有两个氮原子中心, 其中一端带有正 电荷的氮原子通过共轭链烯烃与另一端的氮原子相连, 形成通过共轭链烯烃 “推-拉” 电子的骨架结构, 共轭链

\section{一般由奇数个碳原子组成 ${ }^{[12]}$.}

噻唑橙类染料是由 Lee 等 ${ }^{[13]}$ 发现的一类用于网状 红细胞分析的荧光性能优良的菁染料, 该类染料在游离 状态下几乎无苂光, 而与核酸分子结合后其荧光显著增 强，在荧光检测时可以避开染料自身的荧光干扰，极大 地提高了检测灵敏度, 已成为生物学领域及化学分析领 域学者们研究的热点 ${ }^{[14 ~ 17]}$. 噻唑橙类染料母体一般含 有苯并噻唑和吡啶环或喹啉环，两端杂环通过亚甲基链 连接起来构成共轭体系. 亚甲基链的长度, 不同染料的 吸收及苂光发射光谱波长范围不同. 同时杂环上取代基

\footnotetext{
*E-mail: chenxiuying1975@163.com

Received January 9, 2012; revised April 23, 2012; published online May 11, 2012.

Project supported by the National Natural Science Foundation of China (No. 20706026) and the Fundamental Research Funds for the Central Universities (Nos. JUSRP211A12, JUSRP21110).

国家自然科学基金(No.20706026)、中央高校基本科研业务费专项资金(Nos. JUSRP211A12, JUSRP21110)资助项目.
} 
不同对染料的光谱性质及光稳定性能具有较大影响, 而 对该类染料取代基与光稳定性之间关系的研究文献报 道较少. 我们已经报道了近红外菁染料的的杂环取代基 对染料光稳定性的影响 ${ }^{[18]}$, 本文以苯并噻唑为原料, 合 成了 4 种噻唑环和喹啉环上分别含有不同取代基甲基、 苠基、辛烷基和羟乙基的不对称三次甲基菁染料, 研究 了取代基对染料光谱性质和光稳定性的影响. 合成的染 料分子结构如 Scheme 1 所示.

\section{1 实验部分}

\section{1 仪器和试剂}

紫外-可见吸收光谱在北京普析通用仪器有限责任 公司 TU-1901 型双光束紫外-可见分光光度计上测得; 荧光光谱仪采用美国 Varian 公司 Cary Eclipse 型苂光光 谱仪; ${ }^{1} \mathrm{H}$ NMR 核磁数据在德国 Bruker 公司 AVANCEIII 型 $400 \mathrm{MHz}$ 全数字化核磁共振谱仪测得; 电喷雾质 谱数据在美国 Waters 公司 Maldi Synapt Q-Tof MS 质谱 仪测得. 高分辨质谱在 MICROMASS HPLC-Q-Tof MS 质谱仪上测得. 循环伏安实验在上海辰华 CHI660D 电 化学工作站上测得. 工作电极为玻碳电极, 参比电极为 饱和甘录电极, 对电极为铂丝电极, 扫描速率是 100 $\mathrm{mV} / \mathrm{s}$, 染料浓度为 $1 \times 10^{-4} \mathrm{~mol} / \mathrm{L}$ 乙腈溶液, 电解质为 氯化锂. 实验用水为二次去离子水. 其他试剂如未说明 均为国产分析纯或化学纯试剂, 如未说明均直接应用而 未经过任何处理.

\section{2 染料的合成及表征}

染料的合成参考文献[19].
1.2.1 中间体 2,3-二甲基苯并噻唑对甲基苯磺酸盐(2) 的合成

将 $7.4 \mathrm{~g}$ 中间体 1 (50 mmol)和 $8 \mathrm{~mL}$ 对甲苯磺酸甲 酯(53 mmol)加入到 $10 \mathrm{~mL}$ 乙酸乙酯中 $70{ }^{\circ} \mathrm{C}$ 加热搅拌回 流 $3 \mathrm{~h}$. 冷却, 过滤后得到白色粉末. 剩余母液继续加热 反应，过滤后收集固体粉末，共得到白色固体 $10 \mathrm{~g}$, 粗 收率 $60 \%$, 产品未纯化直接做下一步反应.

1.2.2 中间体 2-甲基-3-苯甲基苯并噻唑溴盐(3)的合 成

将 $3.7 \mathrm{~g}$ 中间体 1 (25 mmol)和 $4.3 \mathrm{~g}$ 溴化苠 $(25$ $\mathrm{mmol}$ )加入到 $5 \mathrm{~mL}$ 乙酸乙酯中 $70{ }^{\circ} \mathrm{C}$ 加热搅拌回流 $2 \mathrm{~h}$. 冷却，过滤沉淀，得到白色固体 $7.2 \mathrm{~g}$, 粗收率 $90 \%$, 产 品未纯化直接做下一步反应.

1.2.3中间体 3-甲基-2-(2-苯胺基乙烯基)苯并噻唑对 甲基苯磺酸盐(4)和溴化-3-苯甲基-2-(2-苯胺基乙烯 基)苯并噻唑盐(5)的合成

将中间体 2 或 3 (10 mmol) 溶于 $10 \mathrm{~mL}$ 醋酸中, 再加 入 $N, N$-二苯基甲脒 $3 \mathrm{~g}(15 \mathrm{mmol}), 70{ }^{\circ} \mathrm{C}$ 加热搅拌, TLC 跟踪. 反应完后冷却, $300 \mathrm{~mL}$ 正己烷洗涤, 析出固体. 过滤得黄色固体 $2.5 \mathrm{~g}$, 粗收率分别为 $60 \%, 85 \%$, 产品 未经纯化直接做下一步反应.

1.2.4中间体碘化-4-甲基-1-辛基喹啉季铵盐(6)的合 成

将 4-甲基喹啉 $1.43 \mathrm{~g}$ (10 mmol), 1-碘辛烷 $2.4 \mathrm{~g}$ (10 $\mathrm{mmol}$ )和 1,4-二氧六环 $20 \mathrm{~mL}$ 依次加入到 $50 \mathrm{~mL}$ 单口烧 瓶中, 加热回流, TLC 跟踪, 反应完全后静置分层, 分

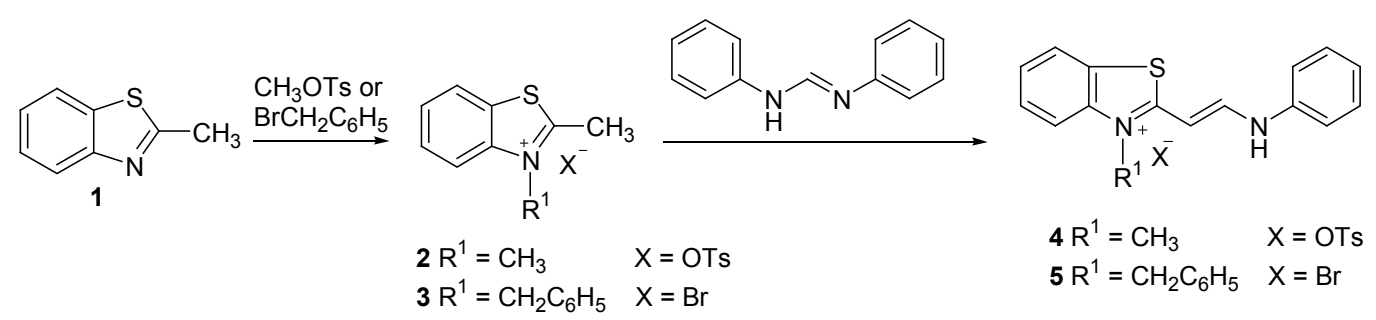<smiles>Cc1ccnc2ccccc12</smiles><smiles>[R]N([R])c1cccc2c(C)cccc12</smiles>

\begin{tabular}{c}
$\mathbf{4}$ or 5 \\
$\underset{\text { paBF }}{4}$ \\
\hline pyridine, reflux
\end{tabular}<smiles>[R]N1C=C/C(=C\C=C\c2sc3ccccc3[n+]2[R7])c2ccccc21</smiles>

$6 \mathrm{R}^{2}=\left(\mathrm{CH}_{2}\right)_{7} \mathrm{CH}_{3} \quad \mathrm{X}=1$ $7 \mathrm{R}_{2}=\mathrm{CH}_{2} \mathrm{CH}_{2} \mathrm{OH} \quad \mathrm{X}=\mathrm{Br}$

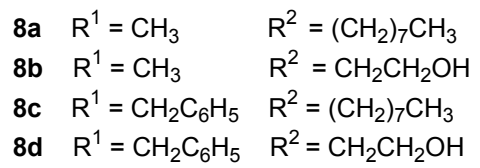

Scheme 1 
去上层溶剂, 剩余液体经柱色谱分离纯化得黄色液体 $2.3 \mathrm{~g}$, 收率 $60 \%$.

1.2.5 中间体溴化 4-甲基-1-(2-差乙基)喹啉季铵盐 (7)的合成

将 4-甲基喹啉 $1.43 \mathrm{~g}(10 \mathrm{mmol})$ 和 2-溴乙醇 $1.23 \mathrm{~g}$ $(10 \mathrm{mmol})$ 依次加入到 $50 \mathrm{~mL}$ 单口烧瓶中, $90{ }^{\circ} \mathrm{C}$ 下暗室 搅拌 $3 \mathrm{~h}$, 冷却过滤, 空气中干燥后得到白色固体 $2.26 \mathrm{~g}$, 未经纯化直接用做下一步反应, 粗收率 $85 \%$.

\subsection{6 染料 $8 \mathbf{a} \sim 8 \mathbf{8}$ 的合成}

称取中间体 6, 7 (1 mmol)分别和中间体 4, 5 (1 $\mathrm{mmol}$ )溶于 $5 \mathrm{~mL}$ 吡啶中, $90{ }^{\circ} \mathrm{C}$ 摚拌下反应, TLC 跟踪, 反应完全后将 $0.160 \mathrm{~g}(1.5 \mathrm{mmol})$ 氟硼酸钠溶于 $1 \mathrm{~mL}$ DMF 中, 加入到反应混合物中, 继续摚拌 $15 \mathrm{~min}$ 反应 完全后冷却, 加入乙醚后析出, 固体抽滤后用乙醇重结 晶得到纯净产物.

3-甲基-2-[3-(1-辛基-4(1H)-喹啉烯基)-1-丙烯基]苯 并噻唑氟硼酸盐(8a): 蓝色固体, 收率 $40 \% .{ }^{1} \mathrm{H}$ NMR $\left(\mathrm{CDCl}_{3}\right) \delta: 0.88 \sim 0.92\left(\mathrm{~m}, 3 \mathrm{H}, \mathrm{CH}_{3}\right), 1.26 \sim 1.90(\mathrm{~m}, 12 \mathrm{H}$, $\left.\mathrm{CH}_{2}\right), 3.71\left(\mathrm{~s}, 3 \mathrm{H}, \mathrm{NCH}_{3}\right), 4.39$ (t, $J=7.6 \mathrm{~Hz}, 2 \mathrm{H}, \mathrm{NCH}_{2}$ ), $6.59(\mathrm{~d}, J=12 \mathrm{~Hz}, 1 \mathrm{H}, \mathrm{C}=\mathrm{CH}), 7.04(\mathrm{~d}, J=12 \mathrm{~Hz}, 1 \mathrm{H}$, $\mathrm{C}=\mathrm{CH}), 7.67(\mathrm{t}, J=12 \mathrm{~Hz}, 1 \mathrm{H}, \mathrm{C}=\mathrm{CH}), 7.16 \sim 8.30(\mathrm{~m}$, $10 \mathrm{H}, \mathrm{ArH}) ; \mathrm{MS} m / z: 429.3[\mathrm{M}]^{+}$. HRMS calcd for $\mathrm{C}_{28} \mathrm{H}_{33} \mathrm{~N}_{2} \mathrm{~S} 429.2359$, found 429.2360 .

3-甲基-2-[3-(1-(2-羊乙基)-4(1H)-喹啉烯基)-1-丙烯 基]苯并噻唑氟硼酸盐(8b): 蓝紫色固体, 收率 $65 \% .{ }^{1} \mathrm{H}$ NMR (DMSO- $\left.d_{6}\right) \delta: 3.73\left(\mathrm{~s}, 3 \mathrm{H}, \mathrm{NCH}_{3}\right), 3.82(\mathrm{t}, J=5.2$ $\left.\mathrm{Hz}, 2 \mathrm{H}, \mathrm{NCH}_{2}\right), 4.64$ (t, $\left.J=4.8 \mathrm{~Hz}, 2 \mathrm{H}, \mathrm{OCH}_{2}\right), 6.47$ (d, $J=12 \mathrm{~Hz}, 1 \mathrm{H}, \mathrm{CH}=\mathrm{C}), 7.14(\mathrm{~d}, J=12 \mathrm{~Hz}, 1 \mathrm{H}, \mathrm{CH}=\mathrm{C})$, $7.28 \sim 8.49(\mathrm{~m}, 10 \mathrm{H}, \mathrm{ArH}), 7.71(\mathrm{t}, J=12 \mathrm{~Hz}, 1 \mathrm{H}, \mathrm{CH}=$ C); MS $m / z: 361.2[\mathrm{M}]^{+}$. HRMS calcd for $\mathrm{C}_{22} \mathrm{H}_{21} \mathrm{~N}_{2} \mathrm{OS}$ 361.1369 , found 361.1363 .

3-苯甲基-2-[3-(1-辛基-4(1H)-喹啉烯基)-1-丙烯基] 苯并噻唑氟硼酸盐 $(8 \mathrm{c})$ : 蓝色固体, 收率 $35 \% .{ }^{1} \mathrm{H}$ NMR $\left(\mathrm{CDCl}_{3}\right) \delta: 0.85 \sim 0.88\left(\mathrm{~m}, 3 \mathrm{H}, \mathrm{CH}_{3}\right), 4.51(\mathrm{t}, J=8 \mathrm{~Hz}, 2 \mathrm{H}$, $\left.\mathrm{NCH}_{2}\right), 1.25 \sim 1.94\left(\mathrm{~m}, 12 \mathrm{H},\left(\mathrm{CH}_{2}\right)_{6}\right), 5.35(\mathrm{~s}, 2 \mathrm{H}$, $\left.\mathrm{CH}_{2} \mathrm{C}_{6} \mathrm{H}_{5}\right), 6.31(\mathrm{~d}, J=12 \mathrm{~Hz}, 1 \mathrm{H}, \mathrm{C}=\mathrm{CH}), 6.70(\mathrm{~d}, J=8$ $\mathrm{Hz}, 1 \mathrm{H}, \mathrm{ArH}), 6.86(\mathrm{~d}, J=12 \mathrm{~Hz}, 1 \mathrm{H}, \mathrm{C}=\mathrm{CH}), 7.06(\mathrm{~d}$, $J=8 \mathrm{~Hz}, 1 \mathrm{H}, \operatorname{ArH}), 7.94(\mathrm{t}, J=12 \mathrm{~Hz}, 1 \mathrm{H}, \mathrm{C}=\mathrm{CH}$ ), 7.14 7.97 (m, 13H, ArH), 8.28 (d, $J=8 \mathrm{~Hz}, 1 \mathrm{H}, \operatorname{ArH})$, $8.73(\mathrm{~d}, J=4 \mathrm{~Hz}, 1 \mathrm{H}, \mathrm{ArH})$; MS $m / z$ : $505[\mathrm{M}]^{+}$. HRMS calcd for $\mathrm{C}_{34} \mathrm{H}_{37} \mathrm{~N}_{2} \mathrm{~S} 505.2672$, found 505.2679.

3-苯甲基-2-[3-(1-(2-差乙基)-4(1H)-喹啉烯基)-1-丙 烯基]苯并噻唑氟硼酸盐(8d): 蓝色固体, 收率 74\%; ${ }^{1} \mathrm{H}$ $\operatorname{NMR}\left(\mathrm{CDCl}_{3}\right) \delta: 3.99\left(\mathrm{t}, J=4 \mathrm{~Hz}, 2 \mathrm{H}, \mathrm{NCH}_{2}\right), 4.67$ (t, $J=$ $\left.4.0 \mathrm{~Hz}, 2 \mathrm{H}, \mathrm{CH}_{2} \mathrm{OH}\right), 5.50$ (s, $\left.2 \mathrm{H}, \mathrm{CH}_{2} \mathrm{C}_{6} \mathrm{H}_{5}\right), 6.42$ (d, $J=$
$12 \mathrm{~Hz}, 1 \mathrm{H}, \mathrm{C}=\mathrm{CH}), 7.04(\mathrm{~d}, J=12 \mathrm{~Hz}, 1 \mathrm{H}, \mathrm{C}=\mathrm{CH}), 7.69$ (t, $J=12 \mathrm{~Hz}, 1 \mathrm{H}, \mathrm{C}=\mathrm{CH}), 7.34 \sim 7.94(\mathrm{~m}, 11 \mathrm{H}, \mathrm{ArH})$, $8.06(\mathrm{~d}, J=8 \mathrm{~Hz}, 1 \mathrm{H}, \mathrm{ArH}), 8.23 \sim 8.17(\mathrm{~m}, 2 \mathrm{H}, \mathrm{ArH})$, 8.47 (d, $J=8 \mathrm{~Hz}, 1 \mathrm{H}, \mathrm{ArH}) ; \mathrm{MS} m / z: 437.2[\mathrm{M}]^{+}$. HRMS calcd for $\mathrm{C}_{28} \mathrm{H}_{25} \mathrm{~N}_{2} \mathrm{OS} 437.1682$, found 437.1685.

\section{3 荧光量子产率}

测量荧光量子产率用罗丹明 B $(\Phi=0.56$, 乙醇) 作 标准样 ${ }^{[20,21]}$, 荧光量子产率公式为:

$$
\Phi_{\mathrm{x}}=\Phi_{\mathrm{s}}\left(F_{\mathrm{x}} / F_{\mathrm{s}}\right)\left(A_{\mathrm{x}} / A_{\mathrm{s}}\right)\left(\lambda_{\mathrm{s}} / \lambda_{\mathrm{x}}\right)\left(n_{\mathrm{x}} / n_{\mathrm{s}}\right)^{2}
$$

式中 $\Phi_{\mathrm{x}}, \Phi_{\mathrm{s}}$ 分别为被测样品和标准物的苂光量子产率; $A_{\mathrm{x}}, A_{\mathrm{s}}$ 分别为被测样品和标准物在激发波长处的吸光度; $n_{\mathrm{x}}, n_{\mathrm{s}}$ 分别为被测样品和标准物所在溶液的折射率; $F_{\mathrm{x}}$, $F_{\mathrm{s}}$ 分别为被测样品和标准物的荧光发射峰的积分面积; $\lambda_{\mathrm{x}}, \lambda_{\mathrm{s}}$ 分别为被测样品的激发波长 $(560 \mathrm{~nm})$ 和标准物的激 发波长 $(470 \mathrm{~nm})$.

\section{4 光降解实验}

将 $1 \mathrm{~cm}$ 厚的石英比色血充满染料的乙醇溶液并保 持密闭, 在 $500 \mathrm{~W}$ 的碘铇灯下照射. 为了除去短波长光 的吸收及照射过程中产生的热量, 在比色典和碘铇灯之 间设置一冷阱, 其中充满 $2 \mathrm{~L}$ 浓度为 $50 \mathrm{~g} / \mathrm{L}$ 的 $\mathrm{NaNO}_{2}$ 溶液, 冷阱宽度为 $15 \mathrm{~cm}$, 碘铇灯与染料溶液间相距 25 $\mathrm{cm}$. 照射过程中尽量保持避光以排除其它因素的影响. 通过测量染料在光照过程中最大吸收值的变化研究染 料的取代基不同对染料光稳定性的影响.

\section{2 结果与讨论}

\section{1 染料的合成}

苯并噻唑不对称三次甲基菁染料由苯并噻唑环和 喹啉环通过三个碳的亚甲基链共轭连接组成, 该类染料 是噻唑橙 ${ }^{[22]}$ 染料的衍生物. 染料的合成以苯并噻唑和 4甲基喹啉为起始原料, 通过和缩合剂 $N, N$-二苯基甲脒反 应得到目标染料. 染料 $8 \mathrm{~b}, 8 \mathrm{~d}$ 的产率比 $8 \mathrm{a}, 8 \mathrm{c}$ 明显提高, 主要是由于 $8 \mathrm{~b}, 8 \mathrm{~d}$ 中喹啉环上引入羟乙基, 比辛烷基取 代染料在反应混合液中具有较差的溶解性, 使其易从溶 液中析出从而利于收集得到固体. 反应温度和反应时间 是影响中间体 4 和 $\mathbf{5}$ 的产率的主要因素, 温度过高和反 应时间延长都会使红色副产物增多，因此控制反应温度 在 $70{ }^{\circ} \mathrm{C}$ 左右, 反应时间在 $1.5 \mathrm{~h}$ 内完成. 4 种染料的纯化 都可以通过乙醇重结晶得到, 降低了染料的合成成本, 使染料更具有实际应用价值.

\section{2 染料的吸收光谱和荧光光谱}

4 种染料在乙醇中的紫外一可见吸收光谱和荧光发 射光谱形状基本相同, 光谱性质见表 1 所示. 图 1 是染 
料 $8 \mathbf{a}$ 在乙醇中经归一化后的吸收和荧光发射光谱. 从 图中可以看出, 染料 $8 \mathbf{a}$ 的最大吸收值在 $629 \mathrm{~nm}$, 最大 苂光发射值在 $662 \mathrm{~nm}$. 染料具有较大的摩尔消光系数 $\left[>10^{5} \mathrm{~L} /(\mathrm{mol} \cdot \mathrm{cm})\right]$. 在溶液中染料基本无苂光, 苂光量 子产率较低, 在 $0.011 \sim 0.023$ 之间, 是由于亚甲基桥链 两端的苯并噻唑(头部)和喹啉环(尾部)之间的自由旋转 造成的 ${ }^{[23 ~ 26]}$. 苠基取代染料 8c, 8d 其 Stokes 位移值比其 它 2 种甲基取代的染料 $\mathbf{8 a}, \mathbf{8 b}$ 的 Stokes 位移大 $3 \sim 4 \mathrm{~nm}$, 可能是由于苠基取代导致由激发态回到基态苂光发射 过程中产生更大的能量损失 ${ }^{[27]}$.

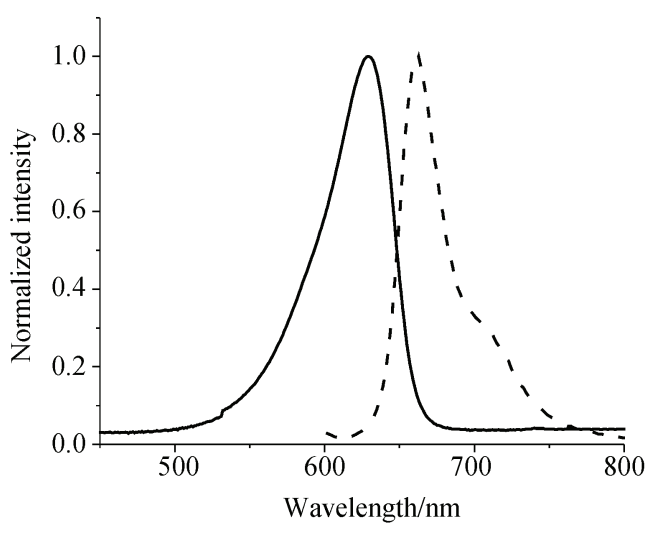

图 1 染料 $8 \mathrm{a}$ 在乙醇中的吸收和荧光光谱

Figure 1 Absorption and fluorescent spectra of dye 8a

表 1 染料在乙醇中的光谱性质

Table 1 Spectral properties of the dyes in ethanol

\begin{tabular}{lccccc}
\hline Dye & $\lambda_{\text {abs }} / \mathrm{nm}$ & $\lambda_{\text {em }} / \mathrm{nm}$ & $\begin{array}{c}\text { Stokes shift/ } \\
\mathrm{nm}\end{array}$ & $\begin{array}{c}\varepsilon /\left(10^{5} \mathrm{~L}^{-\mathrm{mol}^{-1}}\right. \\
\left.\mathrm{cm}^{-1}\right)\end{array}$ & $\Phi$ \\
\hline $\mathbf{8 a}$ & 629 & 662 & 33 & 1.01 & 0.011 \\
$\mathbf{8 b}$ & 631 & 662 & 31 & 1.28 & 0.016 \\
$\mathbf{8 c}$ & 630 & 666 & 36 & 1.61 & 0.014 \\
$\mathbf{8 d}$ & 628 & 664 & 36 & 1.28 & 0.023 \\
\hline
\end{tabular}

\section{3 染料的光降解}

染料应用于荧光检测的前提是染料必须具有高的 光稳定性. 菁染料发生氧化反应是在共轭亚甲基链上与 活性氧如: 单线态氧、超氧负离子自由基等结合的行 为 ${ }^{[28]}$. 为了得到这一类染料的光降解速率常数, 我们对 比研究了染料在乙醇中的光降解性质. 光降解实验在 1 $\mathrm{cm}$ 宽的比色管中进行, 样品溶液用 $500 \mathrm{~W}$ 的碘铇灯照 射, 实验在室温下避光进行. 实验初始, 将 4 种染料的 最大吸收值调至一致, 每隔 $3 \mathrm{~h}$ 测试染料的最大吸收值, 将最大吸收值与光照时间做曲线, 得到染料的光降解反 应过程对比数据, 实验结果见图 2 所示. 光照 $9 \mathrm{~h}$ 后, 染 料 $\mathbf{8 a} \sim 8 \mathbf{d}$ 分别产生了 $30 \%, 49 \%, 14 \%$ 和 $29 \%$ 的光分解, 其中染料 $8 \mathrm{c}$ 降解速率最慢, 说明噻唑环上引入茮基、喹 啉环上引入长链辛烷基使染料具有最强的光稳定性. 这 可能是由于茮基的平面结构和辛烷基的长链结构使其

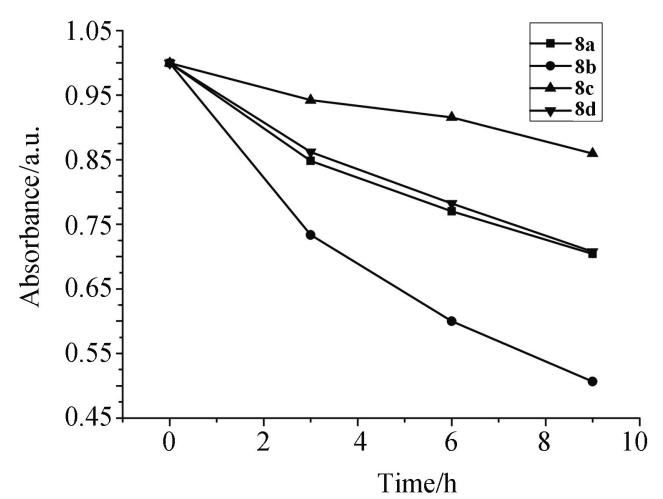

图 2 染料在乙醇中的光降解实验对比

Figure 2 Comparisons on the photodegradation of the dyes in ethanol

空间位阻增大，活性氧不易进攻中间亚甲基链，因而不 易产生光降解.

对于低浓度染料，染料的浓度和最大波长处的吸收 值之间具有线性关系，光降解反应方程可简化如下：

$$
\ln \left(A_{0} / A_{t}\right)=k t
$$

$A_{0}$ 是光照前染料最大波长处的吸收值, $A_{t}$ 是光照中染料 在最大波长处的吸收值, $t$ 是光照反应时间, $k$ 是光降解 反应速率常数, 因而光降解反应速率常数可以通过实验 测到 ${ }^{[29,30]}$. 图 3 所示, 拟合后直线的斜率即为光降解反 应的速率常数, 经过计算, 4 种染料在乙醇中的光降解 速率常数 $k$ 分别是 $6.38 \times 10^{-4}, 12.5 \times 10^{-4}, 2.68 \times 10^{-4}$ 和 $6.30 \times 10^{-4} \mathrm{~mol} \cdot \mathrm{min}^{-1}$. 数据表明，该类染料比七次 甲基菁染料(光降解速率常数约为 $10^{-3} \mathrm{~mol} \cdot \mathrm{min}^{-1}$ ) ${ }^{[18]}$ 具 有更好的光稳定性. 从光降解速率常数 $8 \mathrm{a}>8 \mathrm{c}, 8 \mathrm{~b}>8 \mathrm{~d}$ 可以看出，染料中噻唑环上苄基取代较甲基取代可以增 强染料的光稳定性. 从光降解速率常数 $8 \mathrm{a}<\mathbf{8 b}, \mathbf{8 c}<\mathbf{8 d}$ 可以看出, 染料中喹啉环上被辛烷基取代较羟乙基取代 光稳定性增强. 因此得出结论：两端杂环氮原子上同时 引入长链辛烷基和大体积苠基可以明显增强染料的光

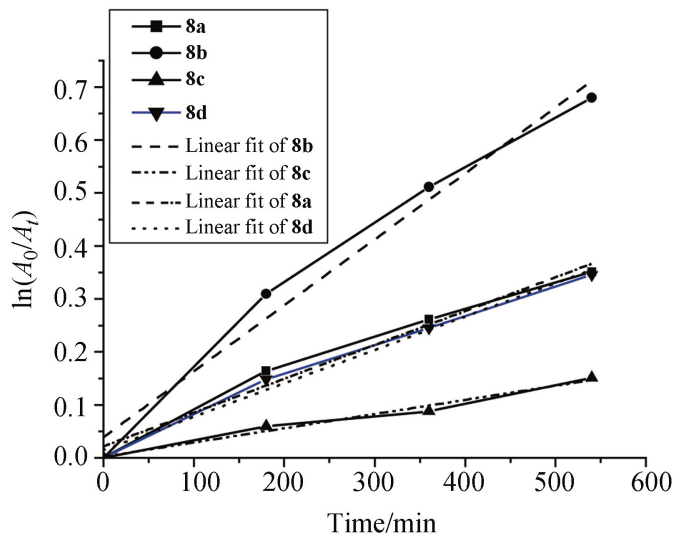

图 3 染料的光降解速率常数

Figure 3 Rate constants of the photodegradation for the dyes 
稳定性. 而羟乙基、甲基则对染料的稳定性不利，可能 是由于大体积的基团可以更有效的阻止活性氧的进攻.

循环伏安实验是一种判断菁染料发生氧化还原反 应难易程度的电化学分析方法 ${ }^{[31,32]}$. 图 4 是染料的循环 伏安曲线, 从循环伏安曲线可以看出, 染料 $8 \mathrm{~b}, 8 \mathrm{~d}$ 的氧 化电位分别为 0.234 和 $0.406 \mathrm{~V}$, 而 $8 \mathbf{a}, 8 \mathrm{c}$ 的氧化峰在 $0.5 \sim 0.8 \mathrm{~V}$ 之间出现一个较宽的峰, 表明喹啉环上辛烷 基取代使染料的氧化电位值增大. 从循环伏安曲线中也 可以看出, 染料光稳定性越高, 其氧化电位值越大.

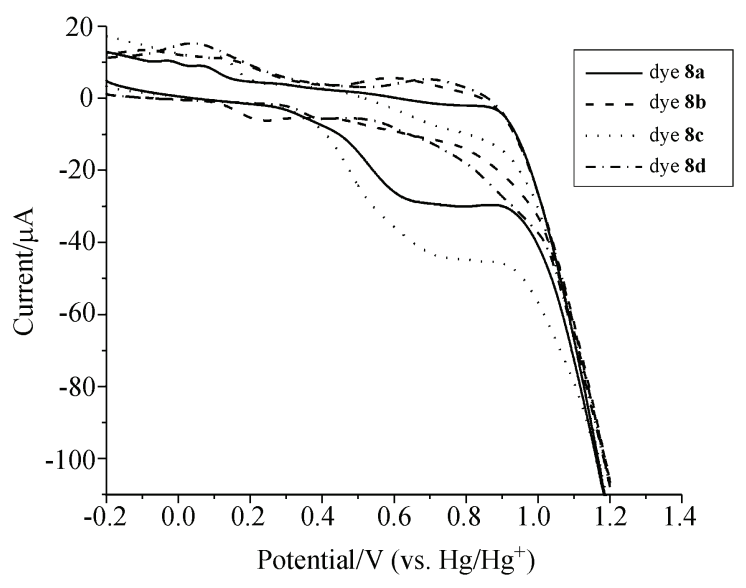

图 4 染料的循环伏安曲线

Figure 4 Cyclic voltammetry curves of the dyes

\section{3 结论}

本文合成并表征了 4 种两端杂环氮原子具有不同取 代基的苯并噻唑不对称三次甲基菁染料, 染料在乙醇中 的最大吸收和苂光发射光谱值分别在 $628 \sim 631$ 和 $662 \sim 666 \mathrm{~nm}$ 之间, 在溶液中染料几乎无苂光. 光降解 实验表明: 染料 $8 \mathbf{a} \sim 8 \mathrm{~d}$ 在乙醇中的光降解速率常数分 别为 $6.38 \times 10^{-4}, 12.5 \times 10^{-4}, 2.68 \times 10^{-4}$ 和 $6.30 \times 10^{-4}$ $\mathrm{mol} \cdot \mathrm{min}^{-1}$; 苯并噻唑环氮原子上引入苠基, 喹啉环上 氮原子引入辛烷基，染料的光稳定性增强. 染料 $8 \mathrm{~b}, 8 \mathrm{~d}$ 的氧化电位分别为 0.234 和 $0.406 \mathrm{~V}$, 喹啉环上辛烷基取 代致使氧化电位峰出现宽峰.

\section{References}

[1] Williams, C. H. G. Trans. R. Soc. Edinburgh 1856, 21, 377.

[2] Chen, C.; Qi, X.; Zhou, B. J. Photochem. Photobiol., A: Chem. 1997, 109, 155.
[3] Beck, W. F.; Sauer, K. J. Phys. Chem. 1992, 96, 4658.

[4] Kietzmann, R.; Willig, F.; Weller, H.; Vogel, R.; Nath, D. N.;Eichberger, R.; Liska, P.; Lehnert, J. Mol. Cryst. Liq. Cryst. 1991, 194, 169.

[5] Kietzmann, R.; Ehret, A.; Spitler, M.; Willig, F. J. Am. Chem. Soc. 1993, 115, 1930.

[6] Mitsuo, K.; Satoshi, A. Chem. Commun. 2004, 988.

[7] Guo, M.; Xu, Y.; Shi, L.; Liang, C.; Wang, L.; Gao, Y.; Sun, S.; Cai, S. J. Photochem. Photobiol. A: Chem. 2005, 171, 231.

[8] Lanzafame, J. M.; Muenter, A. A.; Brumbaugh, D. V. Chem. Phys. 1996, $210,79$.

[9] Lanzafame, J. M.; Min, L.; Miller, R. J. D.; Muenter, A. A.; Parkinson, B. A. Mol. Cryst. Liq. Cryst. 1991, 194, 287.

[10] Zhou, X.; Zhou, J. Anal. Chem. 2004,76, 5302.

[11] Schuler, B.; Pannell, L. K. Bioconjugate Chem. 2002, 13, 1039.

[12] Vasu, N.; Curt, S. C. J. Org. Chem. 1981, 46, 4759.

[13] Lee, L. G.; Chen, C. H.; Chiu, L. A. Cytometry 1986, 7, 508.

[14] Benson, S. C.; Mathies, R. A.; Glazer, A. N. Nucleic Acids Res. 1993, 21, 5720.

[15] Lubitz, I.; Zikich, D.; Kotlyar, A. Biochemistry. 2010, 49, 3567.

[16] Bethge, L.; Singh, I.; Seitz, O. Org. Biomol. Chem. 2010, 8, 2439.

[17] Deligeorgiev, T.; Gadjev, N.; Vasilev, A.; Drexhage, K.-H.; Yarmoluk, S. M. Dyes Pigm. 2007, 72, 28.

[18] Chen, X.; Peng, X.; Cui, A.; Wang, B.; Wang, L.; Zhang, R. J. Photochem. Photobiol., A: Chem. 2006, 181, 79.

[19] Mizukami, T.; Ishiyama, M.; Sakata, T. US 6004816, 1999 [Chem. Abstr. 1998, 160, 78447].

[20] Karstens, T.; Kobs, K. J. Phys. Chem. 1980, 84, 1871.

[21] Velapoldi, R. A.; Tönnesen, H. H. J. Fluoresc. 2004, 14, 465.

[22] Petersen, M.; Hamed, A. A.; Pedersen, E. B.; Jacobsen, J. P. Bioconjugate Chem. 1999, 10, 66.

[23] Stærk, D.; Hamed, A. A.; Pedersen, E. B.; Jacobsen, J. P. Bioconjugate Chem. 1997, 8, 869.

[24] Timcheva, I.; Maximova, V.; Deligeorgiev, T.; Gadjev, N.; Sabnis, R.; Ivanov, I. FEBS Lett. 1997, 405, 141.

[25] Netzel, T. L.; Nafisi, K.; Zhao, M.; Lenhard, J. R.; Johnson, I. J. Phys. Chem. A 1995, 99, 17936.

[26] Jacobsen, J. P.; Pedersen, J. B.; Hansen, L. F.; Wemmer, D. E. Nucleic Acids Res. 1995, 23, 753-760.

[27] Chen, G. Fluorometry, 2nd ed., Science Press, Beijing, 1990, pp. $10 \sim 15$ (in Chinese). (陈国珍, 荧光分析法, 第二版, 科学出版社, 北京, 1990, pp. $10 \sim 15$.)

[28] Song, F.; Peng, X.; Lu, E.; Zhang, R.; Chen, X.; Song, B. J. Photochem. Photobiol., A: Chem. 2004, 168, 53.

[29] Chen, P.; Sun, S.; Hu, Y.; Qian, Z.; Zheng, D. Dyes Pigm. 1999, 41, 227.

[30] Rettig, W.; Strehmel, B.; Schrader, S.; Seifert, H. Applied Fluorescence in Chemistry, Biology and Medicine, Springer, London, 1998, pp. 193 240 .

[31] Lenhard, J. R.; Cameron, A. D. J. Phys. Chem. 1993, 97, 4916.

[32] Kanofsky, J. R.; Sima, P. D. Photochem. Photobiol. 2000, 71, 361. 